\title{
Niedrigzinsen: Ursachen und wirtschaftspolitische Implikationen
}

In Deutschland wird derzeit intensiv über die niedrigen Zinsen und die Rolle der Geldpolitik für die Zinsentwicklung diskutiert. Kritiker der Europäischen Zentralbank (EZB) behaupten, die expansive Geldpolitik sei eine wichtige Ursache der niedrigen Zinsen. Ziel dieser Politik sei eine Entlastung der hoch verschuldeten Ökonomien in Südeuropa. Folge sei eine Umverteilung zulasten vor allem von Sparern mit kleinen und mittleren Einkommen. Außerdem bewirke diese Geldpolitik eine „Zombifizierung“ der europäischen Wirtschaft, also ein schwaches Wirtschaftswachstum, verursacht dadurch, dass Unternehmen ohne nachhaltiges Geschäftsmodell und daher mit niedrigem Produktivitätswachstum durch billige Kredite am Leben gehalten werden. Diese Vorwürfe haben Verteidiger der EZB auf den Plan gerufen, die ihrerseits behaupten, die niedrigen Zinsen seien in erster Linie realwirtschaftlich verursacht, durch hohe Ersparnisse und schwache Nachfrage nach Krediten. Die expansive Geldpolitik der EZB sei eine Voraussetzung dafür, dass die Konjunktur sich nicht noch schwächer entwickle. Von dieser Seite wird häufig gefordert, der deutsche Staat solle sich stärker verschulden, dann würden auch die Zinsen steigen.

\section{Treiber der Zinsentwicklung}

Um die aktuelle Lage an den Kapitalmärkten zu verstehen ist es sinnvoll, die längerfristige Zinsentwicklung zu betrachten. Die realen und nominalen Zinsen folgen bereits seit Jahrzehnten einem Abwärtstrend. Dieser Trend hat lange vor der Gründung der Eurozone begonnen. Folgt man der Analyse von Del Negro et al.,1 dann lag der globale Realzins für „sichere“ und liquide Aktiva historisch über lange Zeiträume bei rund 1,5\%, stieg nach dem Zweiten Weltkrieg vorübergehend auf etwa $3 \%$ an und fing um das Jahr 1980 an, stetig zu sinken. Heute liegt er bei knapp über $0 \%$. Während über viele Jahrzehnte hinweg noch sehr große Unterschiede bei den nationalen Zinstrends zu beobachten waren, trug die Öffnung der weltweiten Kapitalmärkte in den 1980er Jahren dazu bei, dass die meisten Länder seither gleichermaßen vom Zinsrückgang erfasst

(C) Der/die Autor(en) 2020. Open Access: Dieser Artikel wird unter der Creative Commons Namensnennung 4.0 International Lizenz (https:// creativecommons.org/licenses/by/4.0/deed.de) veröffentlicht.

Open Access wird durch die ZBW - Leibniz-Informationszentrum Wirtschaft gefördert.

1 M. Del Negro, D. Giannone, M. P. Giannoni, A. Tambalotti: Global trends in interest rates, in: Journal of International Economics, 118. Jg. (2019), H. C, S. 248-262. wurden und dass die Streuung der nationalen Realzinsen um den globalen Zinstrend aktuell nur sehr gering ist.

Der Rückgang bei den Nominalzinsen war noch ausgeprägter als bei den Realzinsen, weil in den 1980er Jahren auch die Inflationsraten deutlich höher waren als heute. In Deutschland lag die Trendinflationsrate 1980 beispielsweise bei $4 \%$, heute nur noch bei $1,5 \%$. Aus beiden Entwicklungen ergibt sich ein Rückgang des Nominalzinses in Deutschland von 4,5 Prozentpunkten. Heute sollte der Nominalzins demnach in einer konjunkturellen Normalsituation bei rund $2 \%$ liegen. Dass er derzeit eher bei $0 \%$ liegt, kann man also der expansiven Geldpolitik der EZB zurechnen.

Wie kann man den langfristigen Rückgang der Realzinsen erklären? Der Zins ist der Preis für die Überlassung von Kapital. Verschiedene Veränderungen beim Angebot von Kapital und bei der Kapitalnachfrage können den Trend zu immer niedrigeren Realzinsen erklären. Auf der Angebotsseite lassen sich hier die Faktoren Demografie, Einkommensverteilung und außerordentlich hohe Ersparnisse in den Schwellenländern anführen. Der demografische Wandel hat dazu geführt, dass in den letzten Jahrzehnten ein wachsender Anteil der Weltbevölkerung - Menschen mit mittleren oder hohen Einkommen im Alter zwischen 30 und 60 Jahren - Ersparnisse für ihre Altersversorgung bilden möchten. Hinzu kommt, dass umlagefinanzierte soziale Sicherungssysteme bei immer weniger Beitragszahlern und immer mehr Empfängern einen sinkenden Beitrag zur Altersversorgung leisten werden. Da andere Formen der Altersvorsorge wichtiger werden, verstärkt auch das die Sparneigung.

Prof. Dr. Dr. h.c. Clemens Fuest ist Präsident des ifo Instituts und Professor für Volkswirtschaftslehre, Lehrstuhl für Nationalökonomie und Finanzwissenschaft, an der Ludwig-Maximilians-Universität München.

Prof. Dr. Timo Wollmershäuser ist Leiter der Konjunkturforschung und -prognosen am ifo Institut in München. 


\section{Abbildung 1}

Investitionen und Ersparnisse der Entwicklungs- und Schwellenländer

in \% des Bruttoinlandsprodukts
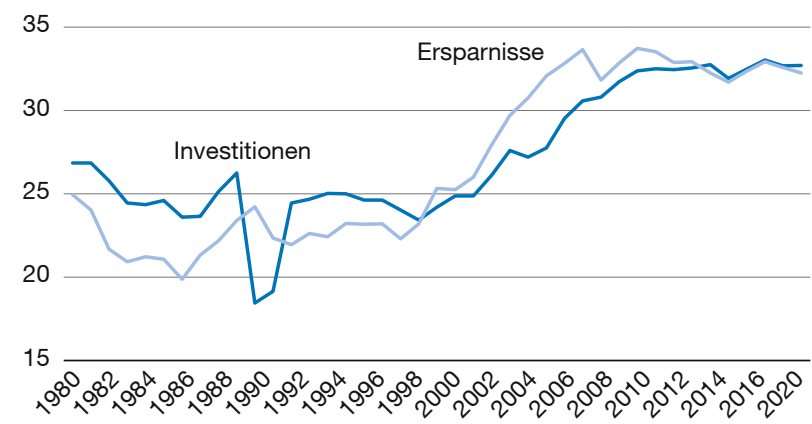

Quelle: International Monetary Fund: World Economic Outlook.

Die Einkommensverteilung hat sich seit den 1980er Jahren weltweit deutlich verändert. Zwar hat die globale Einkommensungleichheit durch den Aufstieg von Schwellenländern wie China und Indien abgenommen. Innerhalb vieler Länder, insbesondere einiger hochentwickelter Industrieländer, allen voran der USA, hat die Einkommensungleichheit aber zugenommen. Da die Sparquote von Haushalten mit dem Einkommen zunimmt, kann hier eine der Ursachen für ein wachsendes Sparangebot liegen.

Darüber hinaus haben die Schwellenländer eine besondere Rolle am globalen Kapitalmarkt gespielt. Man könnte erwarten, dass Schwellenländer im Rahmen ihres wirtschaftlichen Aufholprozesses Kapital aus den entwickelten Ländern benötigen, um die mit dem Aufholprozess verbundenen Investitionen zu finanzieren. Das war jedoch nicht der Fall. Die Entwicklungs- und Schwellenländer haben zwar vor allem in den letzten zwei Jahrzehnten einen Investitionsboom erlebt. Sie haben diese Investitionen aber vollständig aus eigenen Ersparnissen finanziert ( $v g l$. Abbildung 1), ja teilweise überstiegen ihre Ersparnisse sogar die heimischen Investitionen. Das hat dazu beigetragen, das globale Kapitalangebot zu erhöhen und die Zinsen zu senken.

Auf der Kapitalnachfrageseite sind vor allem ein sinkendes Trendwachstum und eine entsprechend sinkende Investitionsnachfrage - überwiegend aus den führenden Industrieländern - anzuführen. Hinzu kommt, dass Investitionsgüter sich in den letzten Jahrzehnten relativ zu Konsumgütern erheblich verbilligt haben. Die Folgen für die Investitionsausgaben hängen von der Preiselastizität der Investitionsnachfrage ab. Rachel und Smith kommen in ihrer Analyse der Zinsentwicklung zu dem Ergebnis, dass diese Relativpreisänderung die Kapitalnachfrage
Abbildung 2

Investitionen und Ersparnisse der Industrieländer in $\%$ des Bruttoinlandsprodukts

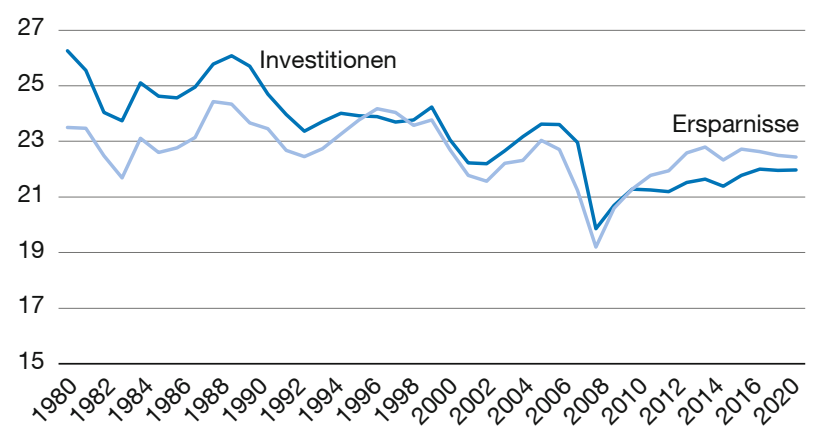

Quelle: International Monetary Fund: World Economic Outlook.

reduziert hat. ${ }^{2}$ Abbildung 2 illustriert die Entwicklung der Investitionen in den Industrieländern seit 1980. Vor allem seit der Finanzkrise haben sie sich schwach entwickelt. Dazu beigetragen haben auch schwache öffentliche Investitionen. Aber ein rückläufiger Trend bestand bereits vorher.

Ein weiterer wichtiger Faktor für den Rückgang des Zinses auf sichere Anlagen ist eine Verschiebung der Nachfrage weg von riskanten und hin zu sicheren Anlagen. Deshalb hat die Renditedifferenz zwischen riskanten und weniger riskanten Anlagen deutlich zugenommen. Dazu beigetragen hat unter anderem die wachsende Nachfrage der Notenbanken nach Staatsanleihen. Caballero et al. weisen zudem darauf hin, dass diese Entwicklung durch einen signifikanten Rückgang des Angebots an sicheren Wertpapieren seit der globalen Finanzkrise verstärkt worden sein dürfte. ${ }^{3}$

Rachel und Smith haben eine quantitative Schätzung zur Rolle verschiedener Faktoren auf der Kapitalangebotsund -nachfrageseite für den Rückgang des globalen Realzinses vorgelegt. Abbildung 3 gibt einen Überblick über das Ergebnis. Nach ihrer Analyse sind die demografisch bedingt verstärkte Sparneigung und sinkende Wachstumserwartungen die wichtigsten Treiber des Zinsrückgangs. Es gibt aber eine Reihe weiterer Einflussfaktoren. Dabei ist zu beachten, dass die Analyse sich auf den "neutralen“ globalen Zinssatz bezieht. Kurzfristige Einflüsse von Konjunktur und Geldpolitik blenden sie aus. Es ist zu betonen,

2 R. Lukasz, T. D. Smith: Are Low Real Interest Rates Here to Stay?, in: International Journal of Central Banking, 13. Jg. (2017), H. 3, S. 1-42.

3 R. J. Caballero, E. Farhi, P.-O. Gourinchas: The Safe Assets Shortage Conundrum, in: Journal of Economic Perspectives, 31. Jg. (2017), H. 3, S. 29-46. 
Abbildung 3

Beitrag zum Rückgang des globalen Realzinses

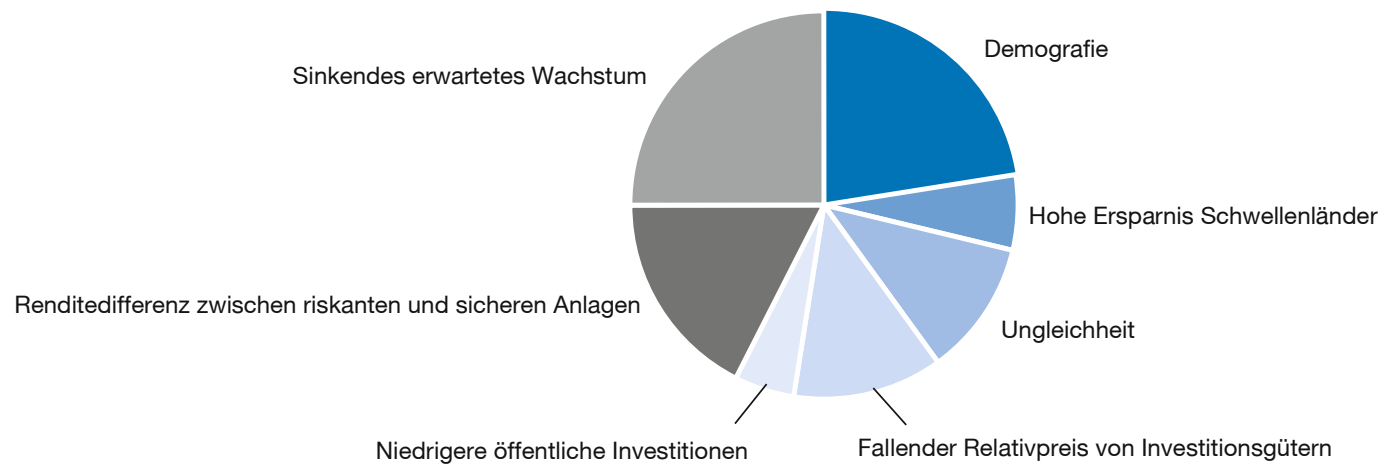

Quelle: R. Lukasz, T. D. Smith: Are Low Real Interest Rates Here to Stay?, in: International Journal of Central Banking, 13. Jg. (2017), H. 3, S. 1-42.

dass diese Quantifizierung auf einer Reihe methodisch bedingter Prämissen beruht, die man nicht teilen muss. Die Bestimmungsfaktoren des neutralen globalen Zinses sind wie dieser Zins selbst nicht direkt beobachtbar. Es wird jedoch deutlich, dass einfache und monokausale Erklärungen dem Phänomen der Niedrigzinsphase nicht gerecht werden.

Die Rolle der Geldpolitik und die Debatte um "Zombifizierung"

Der gesunkene Zinstrend muss auch bei der Einschätzung des Expansionsgrades der Geldpolitik berücksichtigt werden, der aus der Differenz zwischen dem tatsächlichen Zins und seinem konjunkturneutralen Trend abgeleitet werden kann. Nach den oben zitierten Schätzungen beträgt diese Differenz derzeit etwa -2\%. Ob dieser Expansionsgrad angemessen ist, ist schwer zu beurteilen. Auf der einen Seite hat das ifo Institut in seinen Konjunkturanalysen mehrfach darauf hingewiesen, dass selbst unter Berücksichtigung der gesunkenen konjunkturneutralen Trendzinsen die Ausrichtung der EZB-Politik spätestens seit 2017 deutlich expansiver ist als dies in vergleichbaren Situationen in den ersten Jahren der gemeinsamen europäischen Geldpolitik der Fall war. ${ }^{4}$ Demnach hätte die EZB bereits im Laufe des Jahres 2017 Abschied von der Nullzinspolitik nehmen und die Leitzinsen anheben müssen. Auf der anderen Seite hat die expansive EZB-Politik den Anstieg der Verbraucherpreise im Euroraum nur sehr langsam auf ein Niveau beschleunigt, das - abgesehen von energie-

4 T. Wollmershäuser, S. Delrio, M. Göttert, C. Grimme, J. Güntner, C. Krolage, S. Lautenbacher, R. Lehmann, S. Link, W. Nierhaus, M. Reif, R. Šauer, T. Schuler, M. Stöckli, K. Wohlrabe, A. Wolf: ifo Konjunkturprognose Sommer 2018: Gewitterwolken am deutschen Konjunkturhimmel, in: ifo Schnelldienst, 71. Jg. (2018), H. 12, S. 33-87. preisbedingten Preisschüben - selbst am konjunkturellen Hochpunkt Anfang 2018 deutlich unterhalb des Preisstabilitätsziels der EZB lag. Vieles spricht daher dafür, dass sich die geldpolitischen Übertragungskanäle verändert haben und ein und derselbe Impuls heute andere Effekte mit sich bringt als früher.

Man kann wegen der niedrigen Inflationsrate der Meinung sein, dass die EZB derzeit eher das Risiko eingehen sollte, zu spät die Zinsen anzuheben als zu früh. Allerdings ist zu bedenken, dass die Geldpolitik derzeit kaum Möglichkeiten hat, auf einen eventuellen Abschwung mit Zinssenkungen zu reagieren.

Dies stellt die EZB vor große Herausforderungen, da mit der Dauer ihrer Nullzinspolitik auch die negativen Nebenwirkungen zunehmen und die Gefahr steigt, dass sich die reichlich zur Verfügung gestellte Liquidität anderenorts entlädt und zur Bildung von Preisblasen auf den Finanzmärkten beiträgt. Wie eingangs erwähnt wird zudem vor allem in Deutschland immer wieder der Vorwurf erhoben, die lockere Geldpolitik der EZB senke nicht nur den tatsächlichen Zins, sondern sie bewirke selbst einen Rückgang des Zinstrends, weil sie das Produktivitätswachstum verlangsame (Zombifizierung). Es ist nicht auszuschließen, dass durch die niedrigen Zinsen Unternehmen weitergeführt werden, die bei einem höheren Zinsniveau aus dem Markt gedrängt würden. Man kann auch nicht ausschließen, dass dadurch Produktionsfaktoren schneller in produktivere Verwendungen gelenkt werden könnten. Bislang fehlen für diese Zombifizierungsthese allerdings überzeugende empirische Belege. Zinserhöhungen als eine Art „Produktivitätspeitsche" einzusetzen - ein Argument, das auch aus der Debatte über Mindestlöhne bekannt ist - erscheint jedenfalls riskant. Dass die aktuelle Wachstumsschwäche 
dadurch überwunden werden könnte, erscheint nicht plausibel.

\section{Schlussfolgerungen}

Angesichts des seit langer Zeit anhaltenden, globalen Trends zu niedrigeren Zinsen bei international integrierten Finanzmärkten ist es nicht überzeugend, nationale Politiken und nationale ökonomische Entwicklungen als wesentliche Bestimmungsfaktoren der Zinsentwicklung zu identifizieren. Daraus folgt unter anderem, dass die Behauptung, die Aufgabe der „schwarzen Null“ in der deutschen Finanzpolitik erlaube es, wieder zu höheren Zinsen zu kommen, irreführend ist. Der Einfluss der deutschen Finanzpolitik auf das globale Zinsniveau ist zu gering.

Allerdings muss man durchaus fragen, ob es angesichts der niedrigen Zinsen auf deutsche Staatsanleihen unter dem Aspekt der optimalen Gestaltung des öffentlichen Vermögens sinnvoll ist, das Angebot an - aus globaler Sicht sicheren - deutschen Staatsanleihen weiter zu verknappen. Es gibt verschiedene Möglichkeiten, die derzeit sehr guten Verschuldungskonditionen zu nutzen, ohne die Nachhaltigkeit der deutschen Staatsfinanzen zu beeinträchtigen. Das gilt sicherlich für produktive öffentliche Investitionen. Öffentliche Investitionen scheitern derzeit in Deutschland allerdings weniger an Finanzierungsspielräumen als an Genehmigungsverfahren oder am Widerstand der lokalen Bevölkerung gegen Infrastrukturvorhaben. Man könnte die guten Verschuldungskonditionen des deutschen Staates aber auch dazu einsetzen, um ein international diversifiziertes Portfolio an riskanteren, aber auch höher verzinslichen Aktiva zu erwerben, wie es das Konzept des Bürgerfonds vorsieht. ${ }^{5}$ Ein solcher Fonds könnte eingesetzt werden, um die Altersvorsorge gerade von Bevölkerungsgruppen mit niedrigen Einkommen aufzubessern.

Der beobachtete globale und langfristige Trend zu niedrigeren Zinsen impliziert außerdem, dass es wenig überzeugend erscheint, die aktuelle Geldpolitik der EZB oder anderer Notenbanken als Hauptgrund für die niedrigen Zinsen zu benennen. Richtig ist allerdings, dass die Geldpolitik die Entwicklung hin zu niedrigen Zinsen erheblich verstärkt. In der Eurozone kann man davon ausgehen, dass die Zinsen bei einer Normalisierung der Geldpolitik auf rund $2 \%$ steigen würden. Glaubt man allerdings den aktuellen Ankündigungen der EZB, ist es bis zu dieser Normalisierung der Geldpolitik noch ein langer Weg.

5 C. Fuest, C. Hainz, V. Meier, M. Werding: Das Konzept eines deutschen Bürgerfonds, ifo Studie, April 2019. 